\title{
Principais Achados de Colonoscopias Realizadas em Caráter de Urgência e Eletivas
}

\author{
Main Finding on Emergency and Electie Colonoscopies
}

${ }^{1}$ CARLOS HENRIQUE MARQUES DOS SANTOS; ${ }^{2}$ MARCELO DE SOUZA CURY; ${ }^{3}$ FÁBIOTACLA SAAD.

\author{
${ }^{1}$ Titular da Sociedade Brasileira de Coloproctologia e Coordenador do Programa de Residência em Cirurgia Geral do \\ Hospital Regional de Mato Grosso do Sul; ${ }^{2}$ Doutor em gastrotenrologia e endoscopia UNIFESP; membro titular da \\ SOBED e ASGE; ${ }^{3}$ Residente de cirurgia geral do Hospital Regional de Mato Grosso do Sul.
}

\begin{abstract}
SANTOS CHM; CURY MS; SAAD FT. Principais Achados de Colonoscopias Realizadas em Caráter de Urgência e Eletivas. Rev bras Coloproct, 2009;29(1): 083-087.
\end{abstract}

RESUMO: Desde 1960 houve grande avanço tecnológico na observação do aparelho digestivo com o advento dos endoscópios flexíveis, usados inicialmente para o trato digestivo superior e posteriormente também para o inferior, permitindo diagnósticos mais precisos e realização do tratamento em alguns casos. Mesmo em situações de urgência a colonoscopia pode ser utilizada como primeira escolha podendo ser diagnóstica e terapêutica. OBJETIVO: O presente estudo teve por objetivo avaliar quais foram os resultados principais das colonoscopias realizadas no Setor de Endoscopia Digestiva do Hospital Regional de Mato Grosso do Sul. MATERIAIS E MÉTODOS: Foram estudados 290 pacientes submetidos à colonoscopia no Serviço de Endoscopia Digestiva do Hospital Regional do Mato Grosso do Sul, no período de novembro de 2006 a agosto de 2007. Foram analisadas as seguintes variáveis: sexo, idade, caráter de indicação do exame (urgência e eletiva) e diagnóstico. RESULTADOS: A idade média foi de 52 anos, variando de 15 a 92 anos. A maioria dos pacientes era do sexo feminino (56,5\%). Dos 290 exames realizados no período foram constatados $48,5 \%$ (n=141) de colonoscopias com diagnóstico normal. Dentre os anormais houve uma prevalência de doença diverticular dos colos $(n=56)$, seguido de pólipos $(n=37)$, doenças inflamatórias $(n=31)$, neoplasias $(n=18)$ e angiodisplasia $(n=7)$. Os pólipos apresentaram uma maior concentração em colo sigmóide $(n=18)$ seguido pelo colo descendente e reto. As doenças inflamatórias apresentaram como diagnóstico colonoscópio, colite actínica $(n=11)$ seguida por retite actínica (n=7) e retite inespecífica $(n=7)$. Houve um maior número de procedimentos oriundos do ambulatório $(73 \%)$ em contraste com internados (20\%) e provenientes do Pronto Socorro (7\%). CONCLUSÃO: A doença diverticular e os pólipos foram os diagnósticos preponderantes, pois a maior indicação foi a hemorragia digestiva. Uma alta incidência de pólipos em sigmóide demonstrou a importância da retosigmóidoscopia, é um procedimento relativamente seguro e mais barato que a colonoscopia. A baixa incidência de angiodisplasia, resultado discordante da literatura pode ser explicada por uma idade média relativamente baixa, existe mais angiodisplasia no delgado.

Descritores: Colonoscopia, diagnóstico endoscópico, doenças do cólo, polipose, angiodisplasia.

\section{INTRODUÇÃO}

Desde 1960 houve grande avanço tecnológico na observação do aparelho digestivo com o advento dos endoscópios flexíveis, usados inicialmente para o trato digestivo superior e posteriormente também para o inferior, permitindo diagnósticos mais precisos e realização do tratamento em alguns casos ${ }^{1}$.
A retosigmoidoscopia permanece sendo o principal método propedêutico para a investigação das doenças do colo distal e reto, sendo um exame de baixo custo e grande disponibilidade. A colonoscopia é considerada como a técnica de maior acuidade para o diagnóstico de lesões estruturais do colo e, atualmente, é utilizada como a primeira opção propedêutica no diagnóstico da hemorragia digestiva baixa após a retosigmoidoscopia ${ }^{2}$. 
O aperfeiçoamento de tecnologias de videoendoscopia, de informática e o desenvolvimento de recursos auxiliares como a cromoscopia e a magnificação de imagem trouxeram uma grande contribuição para a aplicação mais ampla do método, beneficiando maior número de pacientes ${ }^{1}$.

Em situações de urgência a colonoscopia pode ser extremamente útil para o diagnóstico e tratamento de lesões sangrantes, devendo - se, entretanto, realizar o procedimento completo, com a passagem do aparelho para o íleo terminal. O tratamento pode ser realizado já neste procedimento, uma vez que os avanços na hemostasia endoscópica evoluíram de forma considerável nos últimos anos ${ }^{3}$. MACHICADO e $\mathrm{col}^{3}$. consideram que a colonoscopia em vigência de sangramento ativo é mais eficiente do que quando realizada alguns dias após.

A colonoscopia é útil ainda no diagnóstico e tratamento de diversas outras doenças, tais como a doença diverticular do colo, doenças inflamatórias, pólipos e neoplasias 4 .

Considerando a importância de conhecermos os principais achados colonoscópicos no nosso meio a fim de compararmos estes resultados com a literatura mundial bem como detectar eventuais tendências de mudanças, este estudo teve por objetivo avaliar os resultados colonoscópicos de um Hospital Geral de Mato Grosso do Sul.

\section{OBJETIVO}

Avaliar os resultados das colonoscopias realizadas no Setor de Endoscopia Digestiva do Hospital Regional de Mato Grosso do Sul.

\section{MATERIAIS E MÉTODOS}

Foram estudados 290 pacientes submetidos à colonoscopia no Serviço de Endoscopia Digestiva do Hospital Regional do Mato Grosso do Sul, no período de novembro de 2006 a agosto de 2007.

Os dados foram obtidos dos registros eletrônicos coletados do referido serviço.

Foram analisadas as seguintes variáveis:

1. Sexo.

2. Idade.

3. Caráter de indicação do exame (urgência e eletiva)

4. Diagnóstico.

5. Topografia das lesões.

\section{RESULTADOS}

A maioria dos pacientes era do sexo feminino $(56,5 \%)$ conforme se observa na tabela 1 . A idade média foi de 52 anos, variando de 15 a 92 anos.

Pouco mais da metade dos pacientes não apresentaram alterações à colonoscopia (tabela 2).

Dentre os exames alterados houve uma maior predominância de pacientes com diagnóstico de doença diverticular seguido por pólipos (gráfico 1).

Os pólipos foram um dos principais achados, encontrados mais frequentemente no colo esquerdo e reto (gráfico 2).

Dentre os casos de doença inflamatória intestinal foi encontrado um maior número de colite inespecífica, seguido de retite actínica e retite inespecífica, como demonstrado no gráfico 3.

A distribuição dos pacientes quanto ao caráter de urgência ou não, está demonstrada na tabela 3 . Houve um maior número de pacientes ambulatoriais.

\section{DISCUSSÃO}

A primeira colonoscopia total realizada com sucesso utilizando fibra óptica foi reportada em 1966

Tabela 1 - Distribuição por sexo dos pacientes submetidos à colonoscopia.

\begin{tabular}{lcc}
\hline Sexo & Número de pacientes & \% \\
\hline Feminino & 164 & 56,5 \\
Masculino & 126 & 43,5 \\
Total & 290 & 100 \\
\hline
\end{tabular}

Tabela 2 - Resultados das colonoscopias.

\begin{tabular}{lcc}
\hline Diagnóstico & Total & \% \\
\hline Colonoscopia Normal & 141 & 48,5 \\
Colonoscopia Anormal & 149 & 51,5 \\
\hline
\end{tabular}

Tabela 3 - Distribuição dos exames por origem da solicitação.

\begin{tabular}{lr}
\hline Procedência & \% \\
\hline Ambulatório & 73 \\
Enfermaria & 20 \\
Pronto Socorro & 7 \\
\hline
\end{tabular}




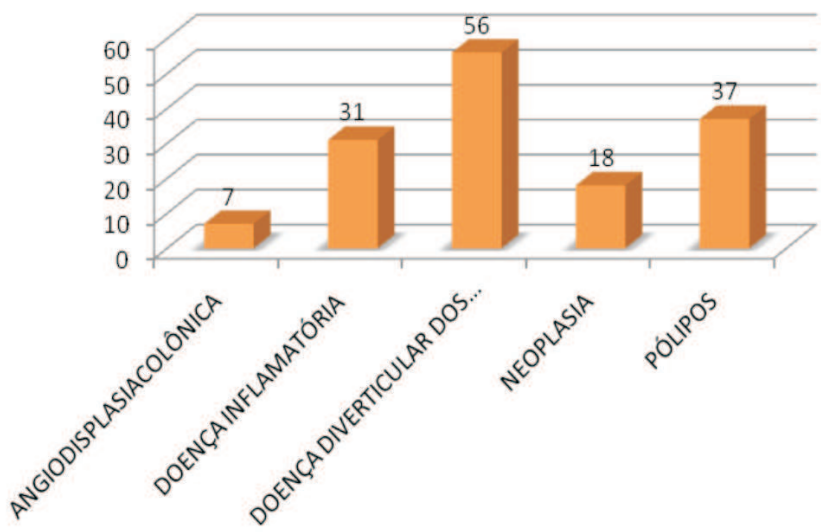

Gráfico 1 - Principais diagnósticos encontrados nas colonoscopias.

por OVERHOLT e POLLARD ${ }^{5}$. A partir de então, com o aperfeiçoamento do método e melhor desenvolvimento técnico, esse exame despontou como o principal meio de avaliação do colo, sendo mais sensível do que o exame radiológico e oferecendo várias opções terapêuticas ${ }^{1}$.

O sangramento intestinal baixo não é uma entidade isolada, mas significa uma gama de diagnósticos que podem estar presentes. A doença diverticular é a causa mais comum seguida em frequência decrescente por pólipo, câncer, doença inflamatória e angiodisplasia ${ }^{6}$.

No presente estudo houve um maior número de exames realizados em mulheres, de forma semelhante ao que se observa na literatura, assim como em relação à idade média de realização da colonoscopia ${ }^{2}$.

O sangramento retal, anemia e alteração do hábito intestinal são as principais indicações para a realização da colonoscopia ${ }^{1}$. Uma vez que existem muitas causas para estas manifestações, tais como doença hemorroidária, doenças funcionais, úlcera péptica, etc., justificam-se assim um grande número de colonoscopias com achados normais, fato também observado no presente estudo com $48,5 \%$ de exames sem alterações. Outras indicações como história familiar de câncer colorretal, emagrecimento, enema opaco inconclusivo e dor abdominal também são indicações frequentes deste exame.

Devemos lembrar, entretanto, que a disponibilidade do método padrão-ouro para a investigação do intestino grosso pode induzir à realização de anamneses incompletas e indicações excessivas da colonoscopia ${ }^{1}$.

O diagnóstico mais frequente nos exames realizados foi doença diverticular dos colos, encontrada



Gráfico 2 - Distribuição segmentar dos pólipos no colo.

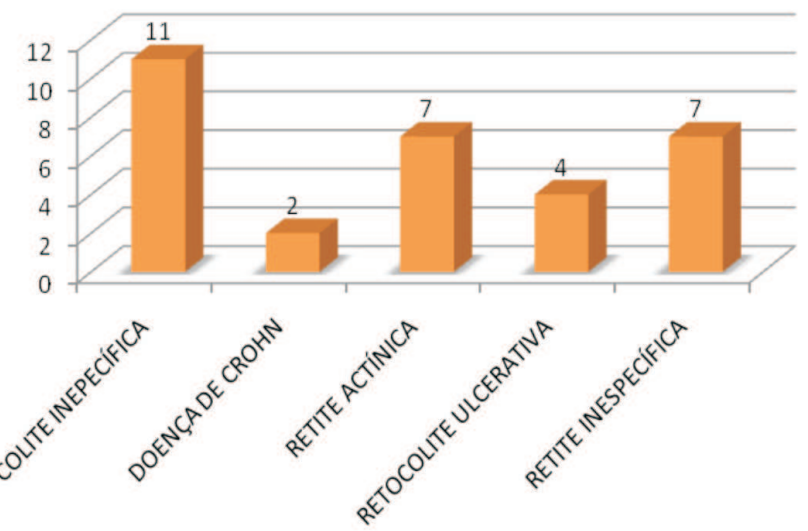

Gráfico 3 - Principais causas de colites.

em $37,5 \%(\mathrm{n}=56)$ dos pacientes, seguido por pólipos em $24,9 \%(n=37)$ deles. Este resultado diverge da literatura que indica pólipos como maior incidência, como o estudo realizado no Reino Unido onde os pólipos foram o achado mais frequente de 9.223 colonoscopias, seguidos pela doença diverticular dos $\operatorname{colos}^{8}$. Essa divergência reflete a sua incidência na faixa etária mais elevada, que foi preponderante neste estudo ${ }^{8}$. Porém, se considerarmos como indicação da colonoscopia apenas a hemorragia digestiva baixa, a doença diverticular dos colos passa a ser a principal causa, seguida de angiodisplasia ${ }^{7}$. Esta divergência pode ser explicada pelo fato de que o serviço de endoscopia do Hospital Regional é referência no estado do Mato Grosso do Sul para tratamento de hemorragia digestiva.

Os pólipos do intestino grosso são lesões de elevada incidência, e, dentre estes, os adenomas são os mais comuns e têm grande relevância pelo seu potencial maligno. Como são assintomáticos ou 
oligossintomáticos na maioria das vezes, é de suma importância a realização de colonoscopia em pacientes acima de 40 anos com história pessoal ou familiar de adenomas ou carcinomas do colo e outros fatores predisponentes ${ }^{9}$.

A retosigmoidoscopia tem se mostrado bastante apropriada para a detecção de pólipos e tumores, embora restrita ao limite natural e óbvio de sua potencialidade, porquanto ela não alcança toda a extensão dos segmentos colônicos. Neste estudo observou-se que dois terços dos pólipos foram encontrados nos segmentos que são acessíveis à retosigmoidoscopia, demonstrando assim a grande importância que este exame ainda apresenta, principalmente em países como o Brasil onde a grande maioria da população encontra grande dificuldade em acesso à colonoscopia 9 .

Foi observado um maior número de colite inespecífica ( $35 \%$ ) dentre as doenças inflamatórias. Isto se deve provavelmente à impossibilidade do endoscopista em afirmar a etiologia da colite sem que tenha acesso a informações mais detalhadas do paciente, baseando-se principalmente nos aspectos e localização das lesões inflamatórias. Naturalmente muitos destes casos poderão ter a etiologia definida pelo médico assistente de posse de maiores informações clínicas, de forma que esta incidência elevada de colite inespecífica não pode ser considerada como uma divergência da literatura mundial ${ }^{10}$.

A angiodisplasia teve uma relativa baixa incidência, o que não vai de acordo com a literatura. A primeira referência encontrada na literatura de hemorra- gia digestiva baixa oriunda de malformações vasculares localizadas no intestino grosso foi feita em $1839^{11}$.

Desde então, muita confusão passou a existir quanto à correta nomenclatura destas alterações vasculares, e sinônimos como hemangiomas, telangiectasias, e ectasias vasculares passaram a ser adotados para descreverem a enfermidade. Tal confusão decorre em parte da multiplicidade de anomalias vasculares que podem acometer o trato digestivo, pois, embora possuam diferentes etiologias, exteriorizam-se clinicamente por sangramento digestivo baixo ${ }^{11}$.

A angiodisplasia atinge ambos os sexos em igual proporção, ocorrendo predominantemente em doentes entre 60 e 70 anos de idade. $\mathrm{O}$ acometimento de indivíduos jovens é achado excepcional ${ }^{12}$, o que pode justificar a baixa incidência de angiodisplasia neste estudo, pois a média de idade foi de 52 anos.

Estima-se que em $15 \%$ dos casos ocorra sangramento maciço. As angiodisplasias correspondem à cerca de $30 \%$ das causas de hemorragia diagnosticadas endoscopicamente ${ }^{13}$.

\section{CONCLUSÃO}

A colonoscopia continua sendo um excelente método diagnóstico e terapêutico para as afecções colorretais. A doença diverticular, seguida de pólipos, foi o diagnóstico preponderante, pois a maior indicação foi a hemorragia digestiva baixa. Uma alta incidência de pólipos em reto e sigmoide demonstrou a importância da retosigmoidoscopia.

ABSTRACT: Since 1960 it there was a great technological breakthrough, including the observation of the digestive system with the advent of flexible endoscopy. Initially used to the upper digestive tract and subsequently to the colon, allowing more accurate diagnoses and, in some cases, the complete treatment. Even in urgency situations, colonoscopy can be used as first choice and work as a diagnostic tool or eve the therapeutic procedure. AIM: This study analyzed the results of colonoscopy held in the Digestive Endoscopy unit at the Hospital Regional Rosa Pedrossian in Mato Grosso do Sul. MATERIALS AND METHODS: It was performed a colonoscopy in 290 patients at the unit of Digestive Endoscopy in the Hospital Regional of Mato Grosso do Sul, in during november 2006 until august 2007. The results were analyzed and verified the following variables: sex, age, solicitation as elective or emergency and diagnosis. RESULTS: The age of the patients ranged from 15 to 92 years old and the mean age was 52 years. Most of the patients were female $(56.5 \%)$. The colonoscopy tests showed that $48.5 \%(n=141)$ were normal. Among the abnormal there were a prevalence of colon diverticulum $(n=56)$, followed by polyps $(n=37)$, inflammatory diseases $(n=31)$, cancer $(n=18)$ and angiodisplasy $(n=7)$. Polyps most usual place were in colorectal sigmoid $(n=18)$ followed by colorectal downward and rectum. The inflammatory diseases were shown as actinic colitis $(n=11)$ followed by actinic proctitte $(n=7)$ and nonspecific proctite $(n=7)$. There was a higher prevalence of procedures from the ambulatory $73 \%$, interns $20 \%$ and from the emergency room $7 \%$. CONCLUSION: The predominant diagnosis was diverticulum disease, followed by polyps. Digestive hemorrhage was the main signal noticed by the doctors who asked for the exam. With a high incidence of polyps, and mostly in sigmoid, just evidenced the how important the retosigmoidoscopy is. It is a relatively safe and cheaper procedure in comparison to the colonoscopy and can be performed during the simple consultation. The low incidence of angiodisplasia could be explained by a relatively low average age, differing from literature.

Key words: Colonoscopy, diagnosis endoscopy, colonic diseases, polyposis, angiodysplasia. 


\section{REFERÊNCIAS}

1. NAHAS SC, MARQUES CFS, ARAÚJO SA, AISAKA AA, NAHAS CSR, PINTO RA, et al. Colonoscopia como método diagnóstico e terapêutico das moléstias do intestino grosso: análise de 2.567 exames. Arq Gastroenterol 2005; 42(2): 7282.

2. SILVA EJ, CÂMARA MAR, GAIDÃO E, ALMEIDA EC. Colonoscopia : Análise crítica de sua indicação. Rev Bras Coloproct 2003; 23(2): 77-81.

3. MACHICADO GA, JENSEN DM. Endoscopic diagnosis and treatment of severe lower gastrointestinal bleeding. Indian Journal of Gastroenterology 2006; 25(1): 43-51.

4. LEVIN TR, ZHAO W, CONELL C, SEEFF LC, MANNIEN DL, SHAPIRO JA, et al. Complications of Colonoscopy in an Integrated Health Care Delivery System. Ann Intern Med 2006; 145: 880-6.

5. OVERHOLT BF, POLLARD HM. Cancer of the colon and rectum. Current procedures for detection and diagnosis. Cancer 1967; 20: 445-50.

6. ORNELLAS AT, ORNELLAS LC, SOUZA AFM, GABURRI PD. Hemorragia Digestiva Aguda Alta e Baixa. In: DANI R, editor. Gastroenterologia Essencial. $2^{\mathrm{a}}$ ed. Rio de Janeiro: Editora Guanabara Koogan S.A.; 2001. p. 3-14.

7. BOWLES CJ, LEICESTER R, ROMAYA C, SWARBRICK E, WILLIAMS CB, EPSTEIN O. A prospective study of colonoscopy practice in the UK today: are we adequately prepared for national colorectal cancer screening tomorrow? Colonoscopy practice in the UK 2004; 53: 277-83.
8. HAWERROTH JA, KURACHI G, JOLY FS, KUPKA RS, DAVILA CP, PINHEIRO RFN. Análise das indicações de colonoscopia no serviço de coloproctologia do HGCR. Rev Bras Coloproct 2007; 27: 23.

9. BEMVENUTI GA, PROLLA JC. Pólipos Intestinais. In: COELHO J, editor. Aparelho Digestivo: clínica e cirúrgica. $3^{\mathrm{a}}$ ed. São Paulo: Editora Atheneu; 2006. p. 1037-44.

10. LAVERY IC. Doenças Inflamatórias Inespecíficas do Intestino Grosso. In: COELHO J, editor. Aparelho Digestivo: clínica e cirúrgica. $3^{\mathrm{a}}$ ed. São Paulo: Editora Atheneu; 2006. p. 94158.

11. KHETERPAL S. Angiodysplasia: a review. J R Soc Méd 1991; 84: 615-8.

12. MARGARIDO NF, CONSOLMAGNO H , PICCININI FILHO L, PRIOLLI DG, MARTINEZ C A R. Angiodisplasia do Ceco na Terceira Década de Vida. Rev Bras Coloproct 2007; 27(2): 202-6.

13. CUTAIT R, AVERBACH M, CORRÊA PAFP. Endoscopia Digestiva Baixa. In: COELHO J, editor. Aparelho Digestivo: clínica e cirúrgica. $3^{\mathrm{a}}$ ed. São Paulo: Editora Atheneu; 2006. p. 174-91.

Endereço para correspondência:

CARLOS HENRIQUE MARQUES DOS SANTOS

Aluisio de Azevedo, 606

Monte Líbano - Campo Grande, MS

CEP 79004-050

Email: chenrriquems@yahoo.com.br Tel: (67) 9221-7174 